\title{
Vision Based Laser Pointer Interaction for Flexible Screens
}

\author{
Nam Woo Kim, Seung Jae Lee, Byung Gook Lee, and Joon Jae Lee \\ Division of Image Contents, School of Design \& IT, \\ San 69-1, Jurye 2-dong, Sasang-gu, Busan, 617-716 \\ $\{$ d5302010, d9003147, lbg, jjlee\}@dongseo.ac.kr
}

\begin{abstract}
In recent years high quality interaction devices have become very popular in our environment. The industries are also currently undergoing rapid change and various technologies have been explored to enable these capabilities. Projection systems using beam projectors and laser pointer became the ubiquitous infrastructure for command technology. Group meetings and other non-desk situations require that people should be able to interact at a distance from a display surface. This paper presents new interaction techniques that use a laser pointer to directly interact with display on a large screen. The camera is subsequently used to detect the position of the pointing device (such as a laser pointer dot) on the screen, allowing the laser pointer to emulate the pointing actions of the mouse. The laser pointer will behave as a active point on the projected display where the user can interact. This vision-based system is augmented with a natural interface that enables the user to interactively refine the suggested rectification. This makes it very easy for users to execute fast and continuous commands. The interaction model developed behaves like a "smart interaction system." The vision based interaction system requires no special hardware and runs on a standard computer.
\end{abstract}

Keywords: Vision-based interaction, determining the mouse interaction, recognize laser spot, camera calibration, nonlinear mapping function.

\section{Introduction}

Interaction with input devices has always been an important part of computer vision. Traditional methods of controlling computer-based interactions require the user to send commands to the computer using either the keyboard or the mouse. But, the ubiquitous environments of today require that people should be able to interact with the display surface from a distance. Much work has been done on the development of laser pointers as interaction devices. The combination of laser pointers and camera as an interaction systems have become an increasingly active topic in the field of Human Computer Interfaces (HCI) and Computer Vision (CV).

Existing systems for accepting user input include expensive electronic whiteboards and pointing devices such as remote mice. Electronic white-boards are not portable and either requires laborious manual calibration and/or forces the use of 
specially coded markers. Remote mice lack of the transparency and immediacy of pointing actions, and suffer from other problems. For instance: infrared mice require the user to point the mouse at a small target; radio mice are subject to interference; mice with long cables are unwieldy.

A single-pointer system for executing more complicated tasks is presented by Olsen and Nielsen [1]. This system has an interaction technique that uses widgets as shown in Figure 1. The selection of widget works well with user interface but requires a feedback procedure. It also projects an alien object on the user interface, which creates distraction.

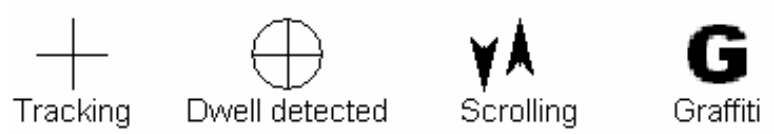

Fig. 1. The feedback is through an echoing cursor and through the selection mechanism for the widgets. There are four cursor modes.

On the other hand, Sukthankar, Stockton and Mullin's self-calibrating cameraassisted presentation interface incorporates virtual buttons that allow user to select function to be performed [3]. However, the addition of virtual buttons requires user to adapt to the user interface [3,4]. Buntarou Shizuki, Takaomi Hisamatsu, Shin Takahashi and Jiro Tanaka use the four edges of a projected screen as the main goals of crossing, which maximizes the goals and thereby maximizes usability. They presented interaction techniques that use a laser pointer for direct manipulation of systems displayed on a large screen. But, the method requires the use of three types of goal crossings. Lines and filled circles depict a laser spot's trajectory on a projected screen and the spot's current position, respectively [7].

This paper presents a robust and efficient laser interaction for imitating mouse function(e.g. move, click, double click and right button) algorithm that allows and enables the user to directly control the interaction at a distance from the computer using a pointing device such as a laser pointer. Our method works intuitively similar to mouse interaction. The laser pointer spot function like a cursor and there is no change to the user interface. The camera can be placed anywhere in the room such that its field of view contains the presentation area. The projector may also be placed anywhere in the room since distortions due to misalignment are automatically corrected regardless external screen such as flexible wall or fabric material by our nonlinear mapping function. It is also an inexpensive technique as it uses only camera and laser pointer. Our system allows user to use the system under a variety lighting conditions, and helps to reduce the amount of image parsing required to find laser position by the order of magnitude.

\section{System Configuration}

An overview of vision based interaction system using laser pointer is shown in Figure 2. The system consists of a laser pointer with a toggle button, a USB2.0 camera to capture the movement of the laser spot projected on the screen, and a computer. A beam projector is used for the projection of display image. The laser pointer's function is to 
interact with the projected image. The camera captures the image projected and obtains the position of laser spot. The computer functions as the processing unit. Software part for the image processing consists of image processing module and coordinate mapping module.

Generally, user must repeatedly go towards the computer to stroke a key in order to flip the slides while doing presentation by using a computer. This distraction to the screen-based explanation interferes with the concentration of both the presenter and the audience. This study investigates and motivates another portable and cheap approach which allows the user to use the laser pointer to control mouse interaction by moving the laser spot and turning the beam on or off. It allows a presenter to point to anywhere on a screen quickly, without concerning the screen size, and the distance of a presenter from the screen.

This makes it very easy for users to run commands, as the vision based interaction enables users to execute fast and continuous commands. System responds correspondingly to various input commands from user.

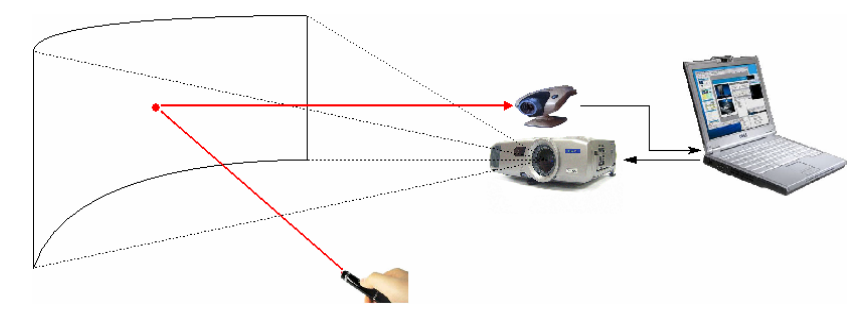

Fig. 2. The vision based interaction system; The portable LCD projector and USB2.0 camera are placed at the front of the screen, while the user can control the mouse function using laser pointer

The software part of vision based system is divided into three modules as shown in figure 3. Initially, the image processing module detects the laser spot, followed by determining the mouse action by the mouse interaction module and then operating system module maps the coordinates. The laser spot module help to find the laser pointer in input image. The mouse interaction module decides mouse event. The operating system module finds out the position.

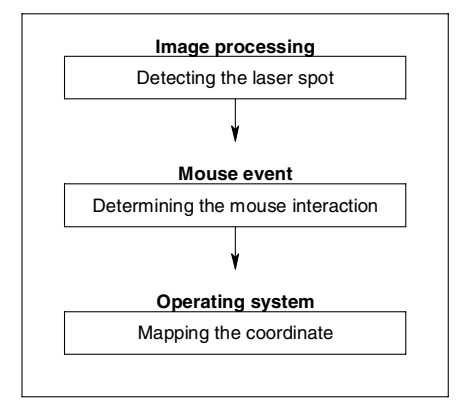

Fig. 3. Flowchart for vision based laser pointer interaction system consisting of image processing, the mouse action and operating system 


\section{Laser Pointer Interaction}

There are three fundamental problems to making this system work.

- Laser pointer detection and recognition

- Interaction function

- Mapping the coordinate

\subsection{Laser Pointer Detection and Recognition}

The robust algorithm used in the system helps to differentiate between noise and the laser pointer. Therefore it is possible to recognize laser spot regardless to the external environment. Laser pointer calculation involves two steps. The color component of RGB color format is first converted into HSI one. The input image $f(x, y)$ is then segmented by equation (1) that is if $f(x, y)$ is in the predefined laser spot region like Figure 4, it is set as " 1 " otherwise " 0 ".

$$
B(x, y)=\left\{\begin{array}{lc}
1, & f(x, y) \in L S \\
0, & \text { otherwise }
\end{array}\right.
$$

where

$$
\begin{gathered}
L S=\left\{(H, S, I) \mid \theta_{1}<H<\theta_{2}, \alpha<S, \beta<I\right\} \\
f_{(x, y)}=\left\{R_{(x, y)}, G_{(x, y)}, B_{(x, y)}\right\} \\
=\left\{H_{(x, y)}, S_{(x, y)}, I_{(x, y)}\right\}
\end{gathered}
$$

The currently commercialized colors (red or green) of laser pointer can be used. Furthermore, if we are using the laser filter (which passes the light having wavelength $630 \mathrm{~nm} \sim 650 \mathrm{~nm}$ in case of red) for spot detection, the efficiency for spot detection is improved by making color range of the laser spot smaller as shown in Figure 4 (b). It is possible to choose the color that user want to detect color for his purpose. Any color can be detected depending on the user requirement. UI controls can recognize each color component value of which users' desire. Then the coordinate mapping module finds the position of spot in projected image, which will be explained in next section. The equipment for laser spot detection consists of a computer attached to a projector and a camera to detect the laser pointer position. The camera used is a USB2.0 camera that can deliver up to 30 frames per second; the algorithms for the image processing involve the color setting module followed by the coordinate mapping module for finding the position.

\subsection{Interaction Function}

This section describes the key ideas regarding the laser pointer based smart control interaction system. The recognizer communicates with interactive system in terms of 


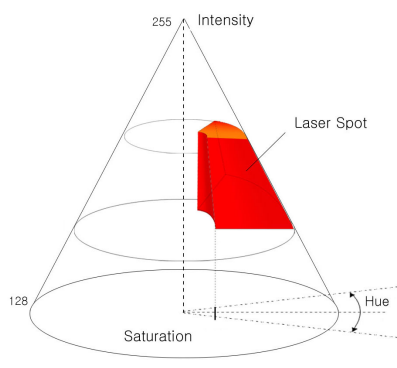

(a)

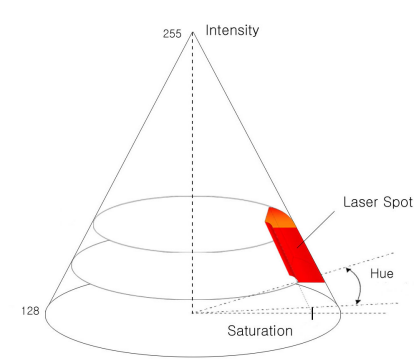

(b)

Fig. 4. The range of laser pointer HSI color model with (a) no band pass filter, (b) band pass filter

four events, namely the mouse move, left button click, double click and right button click. We propose new algorithm that enable the user to directly control the mouse function, at a distance from the computer using just a laser pointer. It is very simple to use and anyone can be trained to use it easily.

Mouse Move. The laser spot detection method has been explained in the upper part. We search for the high intensity red spot. If we cannot find an acceptable spot in that frame, we then search the whole screen in the next frame. Once the laser pointer spot is tracked, we calculate the mapping coordinate of the laser pointer position and return it as the position of the mouse cursor. The fact that cameras, projectors and rooms are all different in their optics and their positioning poses a problem. What is needed is a function that will map a detected laser spot $(x, y)$ position in the camera image to the corresponding position of the mouse cursor in the interactive display. The method is explained in detail at next step. The laser spot is lighted on the display surface when user wants a mouse movement.

The movement is detected when there is difference of coordinate of the laser pointer between two continuous frames. However, we do not want to register this movement as mouse movement because trembling hand can cause minor laser pointer movement. Therefore, a condition to limit the mouse movement only if the laser pointer moves beyond the fixed threshold as follows:

$$
\left\|P_{t}-P_{t-1}\right\|_{L_{1}}>T_{d}
$$

where $P_{t}$ is the position of the laser point at time $T$ (current frame), $P_{t-1}$ is the position of the point in the previous frame and $T_{d}$ is the tolerance given.

Mouse Left Button Click. Left mouse button is pressed and then released once if user wants a mouse left click interaction. Likewise, this action is done using laser pointer by turning the laser pointer on and off in less than a few hundreds of 
milliseconds predefined by setting module. Then laser pointer position $(x, y)$ where the laser pointer light is turned off is recorded.

Mouse Left Button Double Click. Similarly, left mouse button is pressed and released twice if a user wants a mouse left double click interaction. This action is done using laser pointer by turning the laser pointer on and off twice. We consider only the laser pointer position $(x, y)$ where the laser pointer light is turned off for the first time is recorded, the reason of not taking second time the laser pointer is turned on and off is to avoid the laser pointer movement caused by such action twice.

Right Button Click. Mouse right button click action will be activated when the pointer of light is being on for more than a few hundreds of milliseconds on the pointer target. The laser pointer position $(x, y)$ is recorded.

\section{Mapping Image to Screen Coordinate}

In this section we discuss the process of mapping the coordinate of laser pointer position recorded by the camera named as captured image coordinate $(x, y)$ and the mouse cursor position on the projected screen given by window screen coordinate $(u, v)$. This process is done at the background without the knowledge of the user. In other words, given an observed feature at $(x, y)$ in the camera image (e.g., a laser spot), we would like to know the position $(u, v)$ in the display surface corresponding to $(x, y)$. In this coordinate mapping or calibration process, a check board, as shown in Figure 5, is used.

However, in today's virtual reality environment, curve screen is utilized sometimes instead of a flat screen. Unfortunately, the usual method of linear transformation proposed by Sukthankar, Stockton and Mullin[4], could not be applied in such environment. To deal with that, we proposed a quadratic method for nonlinear environment.
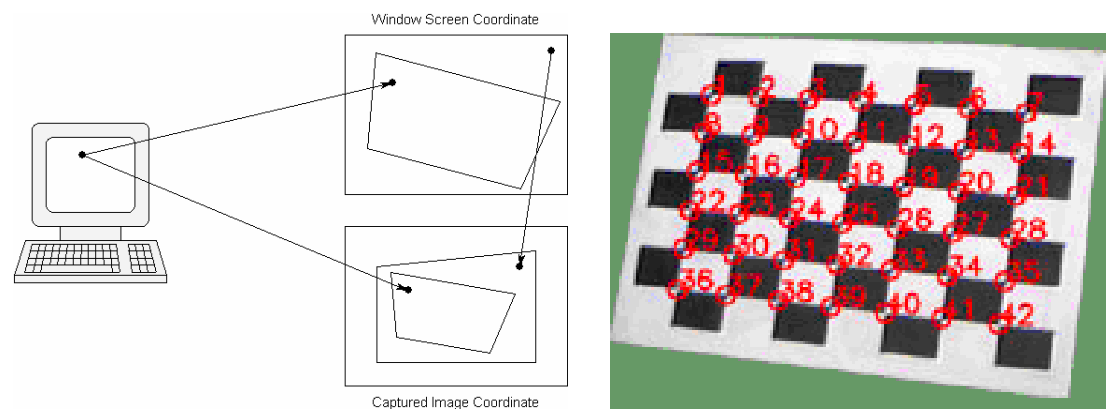

Fig. 5. The relationships between the three frames of reference corresponding to the laptop display (source image frame), camera (camera image frame) and projection screen (projected image frame) 


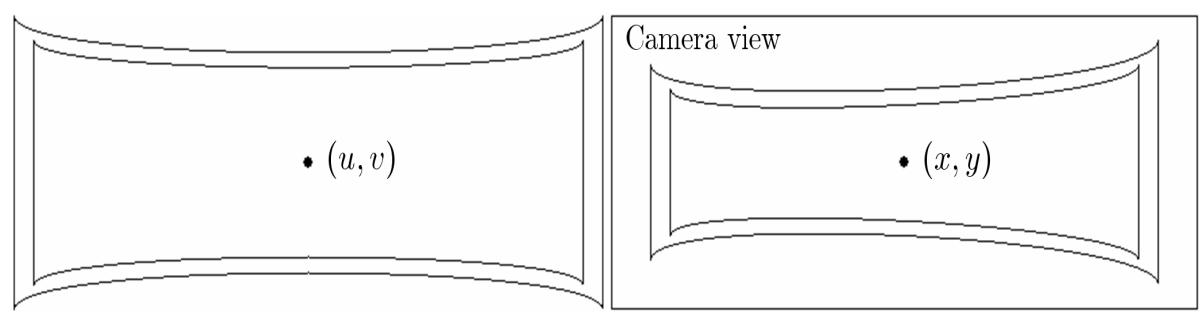

Fig. 6. The application image can be appropriately distorted, using the new mapping algorithm so that it appears nonlinear after projection through a misaligned projector

$$
\begin{aligned}
& u=\frac{p_{1} x^{2}+p_{2} x y+p_{3} y^{2}+p_{4} x+p_{5} y+p_{6}}{p_{13} x^{2}+p_{14} x y+p_{15} y^{2}+p_{16} x+p_{17} y+p_{18}} \\
& v=\frac{p_{7} x^{2}+p_{8} x y+p_{9} y^{2}+p_{10} x+p_{11} y+p_{12}}{p_{13} x^{2}+p_{14} x y+p_{15} y^{2}+p_{16} x+p_{17} y+p_{18}}
\end{aligned}
$$

with eighteen degrees of freedom, $\vec{p}=\left(\begin{array}{llll}p_{1} & p_{2} & \cdots & p_{18}\end{array}\right)$ constrained by $|\vec{p}|=1$. The same transform is more concisely expressed in homogeneous coordinates as:

$$
\left(\begin{array}{c}
u w \\
v w \\
w
\end{array}\right)=\left(\begin{array}{cccccc}
p_{1} & p_{2} & p_{3} & p_{4} & p_{5} & p_{6} \\
p_{7} & p_{8} & p_{9} & p_{10} & p_{11} & p_{12} \\
p_{13} & p_{14} & p_{15} & p_{16} & p_{17} & p_{18}
\end{array}\right)\left(\begin{array}{c}
x^{2} \\
x y \\
y^{2} \\
x \\
y \\
1
\end{array}\right)
$$

$\vec{p}$ can be determined from the obtained corner points from the chess board for calibration.

Given $\mathrm{n}$ feature point matches, $\left\{\left(x_{i}, y_{i}\right),\left(u_{i}, v_{i}\right)\right\}$, let $\mathrm{A}$ be the following $2 n \times 18$ matrix :

$$
\left(\begin{array}{cccccccccccccccccc}
x_{1}^{2} & x_{1} y_{1} & y_{1}{ }^{2} & x_{1} & y_{1} & 1 & 0 & 0 & 0 & 0 & 0 & 0 & -x_{1}^{2} u_{1} & -x_{1} y_{1} u_{1} & -y_{1}{ }^{2} u_{1} & -x_{1} u_{1} & -y_{1} u_{1} & -u_{1} \\
0 & 0 & 0 & 0 & 0 & 0 & x_{1}{ }^{2} & x_{1} y_{1} & y_{1}{ }^{2} & x_{1} & y_{1} & 1 & -x_{1}{ }^{2} v_{1} & -x_{1} y_{1} v_{1} & -y_{1}{ }^{2} v_{1} & -x_{1} v_{1} & -y_{1} v_{1} & -v_{1} \\
\vdots & \vdots & \vdots & \vdots & \vdots & \vdots & \vdots & \vdots & \vdots & \vdots & \vdots & \vdots & \vdots & \vdots & \vdots & \vdots & \vdots & \vdots \\
\vdots & \vdots & \vdots & \vdots & \vdots & \vdots & \vdots & \vdots & \vdots & \vdots & \vdots & \vdots & \vdots & \vdots & \vdots & \vdots & \vdots & \vdots \\
x_{n}^{2} & x_{n} y_{n} & y_{n}{ }^{2} & x_{n} & y_{n} & 1 & 0 & 0 & 0 & 0 & 0 & 0 & -x_{n}{ }^{2} u_{n} & -x_{n} y_{n} u_{n} & -y_{n}{ }^{2} u_{n} & -x_{n} u_{n} & -y_{n} u_{n} & -u_{n} \\
0 & 0 & 0 & 0 & 0 & 0 & x_{n}{ }^{2} & x_{n} y_{n} & y_{n}{ }^{2} & x_{n} & y_{n} & 1 & -x_{n}{ }^{2} v_{n} & -x_{n} y_{n} v_{n} & -y_{n}{ }^{2} v_{n} & -x_{n} v_{n} & -y_{n} v_{n} & -v_{n}
\end{array}\right)
$$

The goal is to find the unit vector $\vec{p}$ that minimizes $|A \vec{p}|$, and this is given by the eigenvector corresponding to the smallest eigen value of $A^{t} A$. And then using (4) we can get mouse position $(u, v)$ of screen coordinate. 


\section{Experimental Results}

The coordinate mapping of distorted screen is done by using check board to identify whether the distortion area of the screen mapped the coordinate correctly. In some occasions, projector may be placed at the side of the room, where it should not get interfered by anything. By the proposed quadratic transformation, 42 corner points of curved screen is mapped with no distortion as shown in Fig. 7 while by linear transformation the rectangular screen appears as a distorted quadrilateral. Next, we have tested the mouse event of mouse move, left click, left double click, and right click to prove our algorithm on flat and curved screen at various angles. This is started from laser spot detection, followed by other functions, as show in Fig. 8. The other events are determined by the time needed by users to perform the action of turning the pointer light on and off. We found out the average time needed is around 0.3 second to get good results. We conclude that our system is suitable for ubiquitous environment where the mouse event can be control by using laser pointer

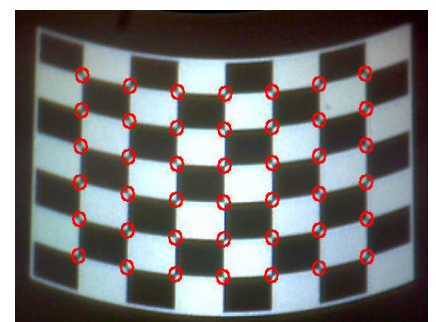

(a)

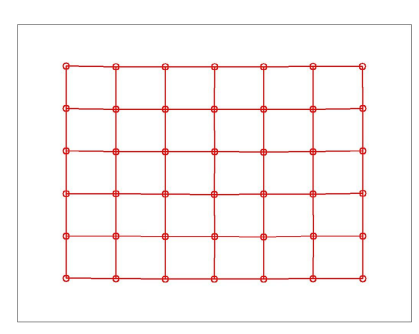

(b)

Fig. 7. Transformation results : (a) shows the curved screen with check board while (b) is the calculated mapping coordinate by nonlinear matrix

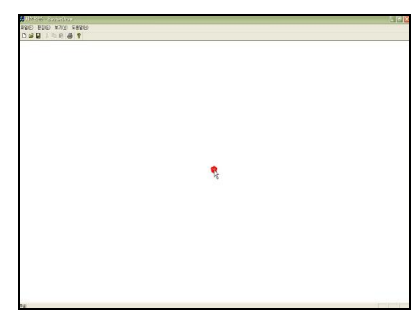

(a)

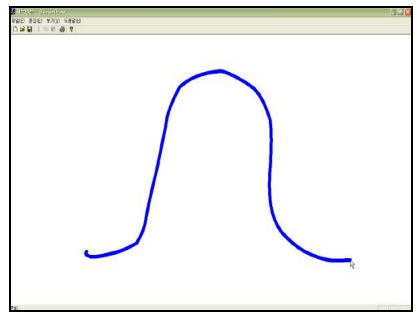

(b)

Fig. 8. Laser spot detection followed by mouse move function according to laser spot movement. (a) is the laser pointer and (b)is the curved made by the mouse pointer as the laser pointer is moved.

\section{Conclusions and Future Works}

Our system provides a better interface for mouse interaction system by allowing users to place portable projectors anywhere in the room, and any distortion produced will 
be automatically rectified. Although many studies have examined the use of laser pointers as input devices, they have suffered from bottlenecks resulting from interaction design. For example, methods proposed by stimulating a button press/release event or began manipulation of GUI parts by leaving the laser spot in one location for a certain period. These methods forced users to wait before executing a command. In contrast, our interaction technique does not involve a delay for the user, enabling quick and continuous command execution. The proposed method presents a robust and efficient laser interaction algorithm that allows the user to directly control the interaction with computer from a distance using a pointing device such as a laser pointer. Additionally, the experimental results show that it also works well on curved screen such as flexible wall or fabric material by our proposed nonlinear mapping function. Furthermore, we want our system to be suitable not only for mouse functions but also scalable to support groups of multiple users, capable of supporting social communication for cooperative tasks.

Acknowledgement. This research was supported by the program for the Training of Graduate Students in Regional Innovation which was conducted by the Ministry of Commerce Industry and Energy of the Korean Government.

\section{References}

1. Olsen Jr., D.R., Nielsen, T.: Laser pointer interaction. Proceedings of the SIGCHI conference on Human factors in computing systems, vol. 17(11) (2001)

2. Ahlborn, B.A., Thompson, D., Kreylos, O.: A practical system for laser pointer interaction on large displays. In: Proceedings of the ACM Virtual Reality Software and Technology, pp. 1006-1009 (2005)

3. Sukthankar, R., Stockton, R.G., Mullin, M.D.: Self-calibrating camera-assisted presentation interface. In: Proceedings of the International Conference on Control, Automation, Robotices and Vision (2000)

4. Sukthankar, R., Stockton, R.G., Mullin, M.D.: Smarter presentations: exploiting homography in camera-projector systems. In: Proceedings of the International Conference on Computer Vision, vol. 1(247) (2001)

5. Sukthankar, R., Stockton, R.G., Mullin, M.D.: Automatic keystone correction for cameraassisted presentation interfaces. In: Proceedings of the International Conference on Multimedia Interfaces. vol. 1948(2000) pp. 607-614 (October 2000)

6. Dekel, A., Kirkpatrick, S.: Room user interface (RUI) design for laser based interaction. submitted to CHI 2005 (2005)

7. Shizuki, B., Hisamatsu, T., Takahashi, S., Tanaka, J.: Laser pointer interaction techniques using peripheral areas of screens. In: Proceedings of the working conference on Advanced visual interfaces (AVI’06), Venezia, Italy. pp. 95-98 (May 23-26, 2006)

8. Oh, J.-Y., Stuerzlinger, W.: Laser Pointers as Collaborative Pointing Devices. Graphics Interface 2002, AK Peters and CHCCS, pp. 141-149 (May 2002)

9. Vogt, F., Wong, J., Po, B.A., Argue, R.S, Fels, S., Booth, K.S.: Exploring collaboration with group pointer interaction. Proceedings of the Computer Graphics International (CGI'04), vol. 00 (June 16-19, 2004)

10. Raskar, R., Brown, M.S., Yang, R., Chen, W.-C., Welch, G., Towles, H., Seales, B., Fuchs, H.: Multi-projector displays using camera-based registraction. In: Proceedings of the conference on Visualization '99: celebrating ten years (IEEE Visualization), San Francisco, California, United States, pp. 161-168 (1999) 